\title{
ESTRÉS VITAL: VARIABLES PSICOLÓGICAS Y SOCIODEMOGRÁFICAS PREDICTORAS DEL MALESTAR EMOCIONAL
}

\author{
LIFE STRESS: PSYCHOLOGICAL AND SOCIO-DEMOGRAPHIC \\ VARIABLES AS PREDICTORS OF EMOTIONAL DISTRESS
}

\author{
BEATRIZ VALLEJO-SÁNCHEZ ${ }^{1}$ Y ANA M. PÉREZ-GARCÍA ${ }^{2}$
}

Cómo referenciar este artículo/How to reference this article:

Vallejo-Sánchez, B., y Pérez-García, A. M. (2016). Estrés vital: variables psicológicas y sociodemográficas predictoras del malestar emocional [Life Stress: Psychological and socio-demographic variables as predictors of emotional distress]. Acción Psicológica, 13(1), 159-178. http://dx.doi.org/10.5944/ap.13.1.16150

\begin{abstract}
Resumen
Introducción: La personalidad y las estrategias de afrontamiento utilizadas ante el estrés juegan un papel importante en el desarrollo de problemas de salud mental. El objetivo de este estudio fue comprobar si estas variables, junto con el estrés percibido y algunas variables sociodemográficas significativas, permitían predecir el nivel de sintomatología ansiosa y depresiva presentada en una muestra de personas con Trastorno Adaptativo (TA), y si las variables predictoras eran las mismas en una muestra control. Método: Se aplicó un protocolo compuesto por cuestionarios de estrés
\end{abstract}

percibido, personalidad, positividad, afrontamiento $\mathrm{y}$ síntomas a un grupo de pacientes que acudieron a una Unidad de Salud Mental, diagnosticados de Trastorno Adaptativo $(N=80)$, y a un grupo control $(N=80)$. Se realizaron análisis de regresión sobre la sintomatología. Resultados: En general, las variables psicológicas y sociodemográficas consideradas contribuyeron en la predicción de la sintomatología, aunque los resultados variaron dependiendo del grupo considerado y del tipo de síntoma. En el grupo control, un mayor neuroticismo, menor positividad, mayor estrés percibido y estar en paro predijeron mayores puntuaciones en depresión, y mayor neuroticismo, estar en paro y ser mujer predijeron mayor ansiedad (no siendo relevante el afrontamiento en ningún caso). En el grupo clínico, la capacidad predictiva de las variables fue menor, especialmente en el caso de la

Correspondencia: Beatriz Vallejo-Sánchez. Unidad de Salud Mental, Hospital Santa Bárbara. Puertollano.

Email: bvallejo@sescam.jccm.es

${ }^{1}$ Unidad de Salud Mental del Hospital Santa Bárbara (Puertollano, Ciudad Real), España.

${ }^{2}$ Universidad Nacional de Educación a Distancia (UNED), Madrid, España. 
ansiedad. Las variables que predijeron mayor sintomatología depresiva fueron ser menos positivo, un mayor uso del afrontamiento de falta de compromiso o evitativo, ser hombre y estar en paro, mientras que en la predicción de la sintomatología ansiosa sólo resultó significativo el neuroticismo. Conclusiones: Las características de personalidad y afrontamiento, el estrés percibido y las características sociodemográficas podrían ser factores de vulnerabilidad a considerar en el desarrollo de estrategias de evaluación, preventivas y de intervención en personas sometidas a estrés vital, aunque probablemente es importante considerar el tipo de muestra en la selección de las estrategias más relevantes.

Palabras clave: personalidad; positividad; afrontamiento; estrés percibido; trastorno adaptativo.

\section{Abstract}

Introduction: Personality and coping used to manage stress play an important role in the development of mental health problems. The aim of this study was to analyse whether these variables, together with perceived stress and some relevant socio-demographic characteristics, could predict the level of anxiety and depressive symptoms presented in people diagnosed with Adjustment Disorder (AD). As well as analysing if the significant predictor variables were the same in the control group. Method: Perceived stress, personality, positivity, coping and symptoms were assessed in a group of patients with Adjustment Disorder who went to a Mental Health Unit $(N=80)$ and a control group $(N=80)$. Regression analyses were carried out to predict symptomatology. Results: Psychological and some socio-demographic variables predicted symptomatology, however, the results were different depending on the group and type of symptom considered. In the control group, higher neuroticism and perceived stress, lower positivity, and unemployment status predicted more depressive symptoms; whereas higher neuroticism, being a female, and unemployment predicted higher anxiety (coping was not relevant in any case). In the clinical group, the predictive capacity of variables was lower, especially in the case of anxiety. Lower positivity, greater use of disengagement or avoidant coping, being a male and unemployment predicted higher depressive symptomatology, whereas only neuroticism predicted symptoms of anxiety. Conclusions: Personality, coping, perceived stress and socio-demographic characteristics could be factors of vulnerability that should be considered in the development of assessment, prevention and treatment strategies with people exposed to life stress. However, the characteristics of the group considered are a key factor in the selection of the most relevant strategy.

Keywords: personality; positivity; coping; perceived stress; adjustment disorder.

\section{Introducción}

La vivencia de situaciones estresantes por parte de las personas a menudo da lugar a dificultades de adaptación importantes y al desarrollo de trastornos mentales que pueden requerir de ayuda o tratamiento especializado. Conocer los factores que influyen en esta relación y en la aparición o no de psicopatología ante el estrés resulta relevante de cara a la elaboración de estrategias de evaluación, preventivas y de intervención con estas personas.

Tras años de investigación, se sabe que tanto el afrontamiento como la personalidad son algunos de los factores más consistentemente asociados al desarrollo de trastornos mentales (Malouff, Thorsteinsson y Schutte, 2005; Mirmics et al., 2013).

En primer lugar, el afrontamiento, entendido como "los esfuerzos, tanto cognitivos como conductuales, que hace el individuo para hacer frente al estrés" (Sandín, 1995, p. 20), juega un papel muy importante, especialmente desde modelos interaccionales que enfatizan la importancia de los factores psicológicos que median entre los agentes estresantes y la respuesta al estrés. En concreto, la teoría transaccional del estrés de Lázarus y Folkman (1984), principal exponente de estos modelos, entiende que el hecho de que un estímulo estresante de lugar a una reacción de estrés depende fundamentalmente 
de la valoración que realiza el sujeto, del estresor y de los propios recursos para afrontarlo. La importancia de los procesos valorativos o subjetivos justificarían la consideración en el estudio de la variable estrés percibido, entendido como la valoración que realiza el sujeto sobre el grado de estrés que le ha producido una situación estresante.

La evaluación cognitiva, a su vez, daría lugar a una determinada reacción al estrés, incluyendo respuestas emocionales, principalmente depresión y ansiedad, y la puesta en marcha de un tipo u otro de afrontamiento, más o menos adaptativo en función de su capacidad para adecuarse a las demandas contextuales (Sandín, 2008). En concreto, y según la clasificación de Carver (1997), podrían diferenciarse dos grandes tipos: afrontamiento de compromiso, en el cual el sujeto intenta activamente manejar la situación o las emociones asociadas a la misma; $\mathrm{y}$ afrontamiento de falta de compromiso, en el que el sujeto se distancia del estresor o de sentimientos relacionados con el mismo. La literatura ha concluido que el primer tipo suele ser más adaptativo y exitoso, y se ha relacionado con una mejor salud mental, mientras que las formas pasivas y de evitación son consideradas menos exitosas y desadaptativas, prediciendo mayores índices de ansiedad y depresión (Carver y Connor-Smith, 2010; Li, Cooper, Bradley, Shulman y Livingston, 2012; Seiffge-Krenke y Klessinger, 2000; Visser et al., 2015).

La personalidad, la otra de las variables consideradas de mayor relevancia en el desarrollo de psicopatología, constituiría una variable que moderaría los diferentes niveles del proceso de estrés, influyendo en los procesos de valoración y en la reacción al estrés, lo que incluiría el desarrollo de síntomas emocionales y el afrontamiento puesto en marcha. Su estudio en los últimos años se ha desarrollado principalmente dentro del modelo de los Cinco Grandes de McCrae y Costa (1991), que considera que existen cinco grandes dimensiones con las que se puede describir la personalidad: Extraversión, Afabilidad o Cordialidad, Tesón o Responsabilidad, Neuroticismo y Apertura mental o Apertura a la experiencia. La investigación ha concluido que el neuroticismo es uno de los predictores más robustos para el desarrollo de psicopatología ante sucesos vitales estresantes (Lahey, 2009; Malouff et al., 2005; Mandelli et al., 2015), relacionán- dose especialmente con síntomas y trastornos depresivos y de ansiedad. Aunque también se han encontrado relaciones con otras dimensiones de personalidad, especialmente con la extraversión (Mirnics et al., 2013; NaragonGainey, Rutter y Brown, 2014), que correlaciona negativamente con problemas ansiosos y depresivos (Lucas y Fujita, 2000; MCrae y Costa, 1991; Watson y Clark, 1992). Las relaciones del resto de dimensiones de personalidad con la psicopatología han sido menos estudiadas, y aunque apuntan a relaciones positivas con la salud mental, especialmente en el caso del tesón y la afabilidad, en general los resultados han sido más débiles e inconsistentes, relacionándose más con trastornos externalizantes frente a los internalizantes -ansiedad y depresión(Kotov, Gamez, Schmidt y Watson, 2010).

Y en cuanto a la dimensión de positividad, un constructo no recogido dentro del modelo anterior y entendido como la propensión a valorar positivamente la vida y las experiencias, parece ser un predictor importante del bienestar y la salud, habiéndose encontrado relaciones negativas con el afecto negativo y la presencia de síntomas depresivos (Caprara et al., 2012), no con ansiedad, aunque por el momento la investigación es escasa.

El estudio conjunto de estas variables psicológicas con otras sociodemográficas está justificado por su relevancia y relación tanto con estas variables (Bermúdez, Teva y Buela-Casal, 2009; McCrae et al., 1999) como con la psicopatología (Altemus, Sarvaiya y Epperson, 2014; Tang, Liu, Liu, Xue y Zhang, 2014; VallejoSánchez y Pérez-García, 2015a).

Por otro lado, existe mucha investigación que relaciona las dimensiones comentadas con el desarrollo de trastornos ansiosos y depresivos, pero apenas se han encontrado trabajos que estudien estas relaciones en muestras con Trastorno Adaptativo (TA), que se trata de un diagnóstico que se realiza con mayor frecuencia incluso que los anteriores cuando una persona pasa por una situación de estrés y desarrolla síntomas emocionales o comportamentales significativos, pero no cumple criterios para trastornos depresivos o de ansiedad más específicos (American Psychiatric Association [APA], 2013). Esta notable escasez de trabajos es probablemente debida a las diferentes controversias existentes en torno a esta catego- 
ría diagnóstica, principalmente por sus criterios vagos y escasamente definidos que en ocasiones dificultan su diferenciación tanto con otros trastornos mentales como con la normalidad (Casey, Dowrick y Wilkinson, 2001). Sin embargo, algunos resultados apuntan a que las variables psicológicas comentadas anteriormente pudieran ser también relevantes en los TA en particular. En concreto, se ha encontrado que en función del nivel de positividad y del estilo de afrontamiento utilizado se puede predecir la presencia de un TA (Vallejo-Sánchez y Pérez-García, 2015a), y además, que los sujetos con TA utilizan más estilos de afrontamiento evitativos o de falta de compromiso, y menos el humor (For-Wey, Fei-Yin y BihChing, 2002; Vallejo-Sánchez y Pérez-García, 2015b), Por tanto, el estudio de estas variables en muestras de sujetos con TA resulta muy relevante de cara a la elaboración de estrategias de evaluación e intervención, así como su comparación con muestras generales o con otras patologías, de cara a comprobar si las anteriores variables de vulnerabilidad (o protección) tienen diferente peso en función del grupo.

Considerando el marco teórico y empírico planteado, nos planteamos como primer objetivo comprobar la capacidad predictiva de las dimensiones de personalidad más relevantes (neuroticismo, extraversión y positividad), de cinco tipos de afrontamiento, del estrés percibido y de algunas variables sociodemográficas significativas sobre el nivel de sintomatología depresiva y ansiosa presentado en una muestra de personas con Trastorno Adaptativo (TA), en comparación con una muestra control. Del anterior objetivo se derivaría la hipótesis general de que las variables estudiadas serían relevantes en la predicción de ambos tipos de síntomas, y varias hipótesis específicas: 1) Se esperaba que el neuroticismo predijera positivamente tanto la sintomatología ansiosa como depresiva, mientras que mayor extraversión y positividad predecirían menor nivel de sintomatología; 2) Las estrategias de afrontamiento de compromiso, humor, búsqueda de apoyo social, y religión, predecirían negativamente ambos tipos de síntomas, mientras que las estrategias de falta de compromiso o evitativas los predecirían positivamente; 3) Se esperaba que un mayor estrés percibido predijese más sintomatología ansiosa y depresiva; 4) En cuanto a las variables sociodemográficas, ser mujer, tener menor edad, menor nivel educativo y estar des- empleado se relacionaría con una mayor sintomatología; y por último, 5) Se esperaba encontrar los mismos resultados en ambas muestras, considerando que previamente no se habían encontrado estudios que realizasen estos mismos análisis.

\section{Método}

\section{Participantes}

El estudio, de tipo transversal y descriptivo, fue realizado con 160 sujetos, divididos en dos grupos, cuyas características sociodemográficas se describen en la Tabla 1.

El grupo clínico estuvo constituido por 80 pacientes que acudieron a una Unidad de Salud Mental (USM) y fueron diagnosticados de TA en base a criterios diagnósticos de la CIE-10 (World Health Organization [WHO], 1992) o del DSM-IV-TR (APA, 2000). Se excluyó a todos aquellos pacientes que presentaban comórbidamente otra psicopatología grave del Eje I o II del DSM-IV-TR, con ayuda de las entrevistas diagnósticas MINI (International Mini Neuropsychiatric Interview; Sheehan et al., 1998; adaptación española de Ferrando et al., 1998) e IPDE (International Personality Disorders Examination; Loranger, 1995; versión española de López-Ibor, Pérez y Rubio, 1996). También se excluyó a los que habían realizado tratamiento psicológico o psiquiátrico en los últimos dos años, así como aquellos cuyas edades no estuviesen comprendidas entre los 18 y 65 años y los que presentasen condiciones que dificultasen la aplicación de los instrumentos de estudio (deterioro cognitivo y/o médico, o nivel educativo o intelectual insuficiente).

El grupo control estuvo formado por 80 personas que habían sufrido en los últimos dos años al menos una situación estresante importante pero que no habían desarrollado psicopatología. En concreto, personas valoradas en la Unidad y dadas de alta por no presentar patología, profesionales del hospital, familiares de éstos últimos y familiares de pacientes de la USM. 


\section{Instrumentos de evaluación y procedimiento}

Para valorar el estrés percibido, se construyó una Escala de sucesos vitales (ESV), consistente en un listado de 40 situaciones estresantes de diferente tipo, extraídas del listado de problemas psicosociales y ambientales que constituye el Eje IV del sistema de evaluación multiaxial del DSM (APA, 2000). El sujeto debía indicar la ocurrencia del estresor en los últimos dos años y el nivel de estrés generado en una escala tipo Likert de cuatro puntos (de $0=$ Ningún estrés a $3=$ Mucho estrés). Se consideró en los análisis la variable de estrés percibido en los sucesos experimentados, que presentó en esta muestra una fiabilidad adecuada $(\alpha=.74)$.

Para medir la personalidad se utilizó el Big Five Inventory-44 (BFI-44; Benet-Martínez y John, 1998), inventario autoaplicado de 44 ítems tipo Likert de cinco puntos (desde $1=$ Muy en desacuerdo, a $5=$ Muy de acuerdo), que evalúa la personalidad según el "Modelo de los Cinco Grandes", y que muestra muy buenas propiedades psicométricas (Benet-Martínez y John, 1998; Rammstedt y John, 2007). En este estudio sólo se consideraron las escalas de Extraversión y Neuroticismo, por su mayor significación en el estudio de la psicopatología, y que mostraron en esta muestra índices de fiabilidad adecuados $(\alpha=.70$ y $\alpha=.84$, respectivamente).

Para medir la orientación positiva se utilizó la Escala de Positividad (Caprara et al., 2012), una escala autoaplicada de 8 ítems tipo Likert de cinco puntos (desde $1=$ Totalmente en desacuerdo, a $5=$ Totalmente de acuerdo) que evalúa el constructo de positividad. En el estudio original se encontró a través de análisis factoriales que presenta una estructura unidimensional, además de buenas propiedades psicométricas (Caprara et al., 2012), aunque con nuestra muestra los índices fueron incluso superiores $(\alpha=.83)$.

Para medir el afrontamiento se utilizó el Brief COPE (Carver, 1997; versión española de Morán, Landero y González, 2010), un inventario autoaplicado de 28 ítems, agrupados en 14 escalas de dos ítems cada una, con formato tipo Likert de cuatro puntos (desde $0=\mathrm{No}$, en absoluto, a 3 =Totalmente) que evalúa el estilo de afrontamiento utilizado ante el estrés. No se aplicaron en este estudio las escalas de consumo de sustancias y de autodistracción, por su comportamiento problemático e inestable. Además, con el fin de aumentar la fiabilidad de la prueba, se mantuvo la configuración original de las escalas de humor y religión que presentaban una alta consistencia, y tal como recomienda su autor (Carver, 1997), las restantes diez escalas se agruparon (mediante la aplicación de un análisis factorial) obteniéndose tres factores: compromiso (que incluye los ocho ítems de las escalas de afrontamiento activo, planificación, reevaluación positiva y aceptación), falta de compromiso (con los ocho ítems de negación, abandono, autoculpa y desahogo) y búsqueda de apoyo social (con los cuatro ítems de apoyo instrumental y apoyo emocional). Las cinco agrupaciones de las estrategias de afrontamiento finalmente consideradas presentaron un índice promedio de consistencia interna de .80 en esta muestra (ver Vallejo-Sánchez y Pérez-García, 2015a, para una descripción más detallada del proceso seguido).

Para la medida de la sintomatología ansiosa se utilizó la adaptación española (Sanz y Navarro, 2003) del Inventario de Ansiedad de Beck (Beck, Brown, Epstein y Steer, 1988). Se trata de un cuestionario autoaplicado, formado por 21 ítems tipo Likert de cuatro puntos (desde $0=$ En absoluto, a 3 =Severamente), que evalúa la presencia y severidad de los síntomas de ansiedad en la última semana en pacientes con trastornos psicológicos y en la población general. Presenta también adecuadas propiedades psicométricas, tanto en el estudio original como en diferentes muestras españolas (Beck, Brown et al., 1988; Sanz, García-Vera y Fortún, 2012; Sanz y Navarro, 2003). En nuestro estudio el índice de consistencia interna fue muy alto $(\alpha=.96)$.

Y por último, los síntomas depresivos se evaluaron a través del la versión española (Vázquez y Sanz, 1997) del Inventario de Depresión de Beck (Beck, Rush, Shaw y Emery, 1979), uno de los cuestionarios autoaplicados más utilizados para la valoración de la depresión. Consta de 21 ítems que valoran diferentes síntomas depresivos, con cuatro opciones de respuesta cada uno (graduadas 
según su intensidad de 0 a 3 ), debiendo el sujeto elegir aquella con la que más se identifique en función de cómo se ha sentido en la última semana. Presenta adecuadas propiedades psicométricas, tanto en su versión original como en la española (Beck, Steer y Garbin, 1988; Vázquez y Sanz, 1997), habiéndose encontrado con nuestra muestra un índice de consistencia interna muy elevado $(\alpha=.94)$.

Todos los sujetos que cumplieron con los criterios de inclusión y firmaron el consentimiento informado para participar fueron evaluados, previa aprobación por parte del Comité de Ética del Hospital, a través de los anteriores instrumentos, que se cumplimentaron en todos los casos en el domicilio.

\section{Análisis de datos}

Se utilizó el programa estadístico SPSS para Windows, versión 19.0 (IBM Corp, 2010). En primer lugar, se comprobó si existían diferencias entre los grupos clínico y control en las variables sociodemográficas medidas utilizando la prueba de $\chi^{2}$ en las variables categóricas, y análisis de varianza (ANOVA) de un factor (grupo) en el caso de la edad.

A continuación, se contrastaron mediante ANOVA las diferencias entre ambos grupos en las variables psicológicas analizadas. Finalmente, para estudiar las variables que predecían los síntomas de ansiedad y de depresión, se llevaron a cabo análisis de regresión lineal múltiple, siguiendo el método Introducir, inicialmente con la muestra total, y posteriormente, con cada una de las muestras (clínica y control) al obtener que la variable grupo era un predictor significativo. En los análisis por grupo, además de las variables sociodemográficas, se tomaron aquellas psicológicas que correlacionaban significativamente con los criterios analizados (los síntomas de ansiedad y de depresión), a partir de las correlaciones bivariadas realizadas. En todos los análisis realizados, el cálculo de la tolerancia y el FIV mostró que no existían efectos de colinealidad significativos entre las variables predictoras (todos los coeficientes de tolerancia fueron superiores en cada caso a $1-\mathrm{R}^{2}$ y los FIV inferiores a 10).

\section{Resultados}

\section{A) Análisis de las características socio- demográficas}

Los resultados de los análisis de las variables sociodemográficas (ver Tabla 1) indicaron que no existían diferencias significativas entre los grupos en la distribución de varones y mujeres $\left(\chi^{2}=1.34, \mathrm{gl}=1, p=.16\right)$ y del estado civil $\left(\chi^{2}=3.98, \mathrm{gl}=3, p=.26\right)$. En ambos grupos había más mujeres que hombres, y estuvieron en su mayoría solteros o casados, siendo muy pocos los separados/divorciados o viudos.

Sin embargo, sí que se encontraron diferencias en las variables situación laboral $\left(\chi^{2}=40.10, \mathrm{gl}=1, p<.001\right) \mathrm{y}$ nivel educativo $\left(\chi^{2}=25.05, \mathrm{gl}=2, p<.001\right)$, siendo casi significativas las diferencias en edad $\left[\mathrm{F}_{(1,158)}=3.49\right.$, $p=.06]$. El grupo clínico fue de media algo más joven que el control, y se encontraba en situación laboral no activa con más frecuencia (en cuanto que había más personas desempleadas o bien dedicadas a estudiar o a las tareas del hogar). Y su nivel de estudios se encontraba entre básico y medio, principalmente, frente al grupo control que en su mayoría tenía un nivel medio de estudios, habiendo en ambos grupos prácticamente la misma proporción de personas con estudios superiores (ver Tabla 1).

Todas las variables sociodemográficas relevantes (sexo) o que resultaron significativamente diferentes entre el grupo clínico y control (situación laboral, y nivel educativo), o próximas a la significación (edad), fueron incluidas en los análisis estadísticos posteriores. 
Tabla 1

Características sociodemográficas de los participantes del estudio

\begin{tabular}{|c|c|c|c|}
\hline & $\begin{array}{l}\text { Grupo clínico } \\
\qquad(N=80)\end{array}$ & $\begin{array}{l}\text { Grupo control } \\
\quad(N=80)\end{array}$ & $\begin{array}{c}\text { Muestra total } \\
\quad(N=160)\end{array}$ \\
\hline Edad [media $(D T)]$ & $36.14(13.47)$ & $39.84(11.53)$ & $37.99(12.63)$ \\
\hline \multicolumn{4}{|l|}{ Sexo [n (\%)] } \\
\hline - Hombres & $25(31.3)$ & $32(40)$ & $57(35.6)$ \\
\hline - Mujeres & $55(68.8)$ & $48(60)$ & $103(64.4)$ \\
\hline \multicolumn{4}{|l|}{ Estado civil [n (\%)] } \\
\hline - Soltero & $36(45)$ & $32(40)$ & $68(42.5)$ \\
\hline - Casado & $34(42.5)$ & $43(53.8)$ & $77(48.1)$ \\
\hline - Separado/Divorciado & $8(10)$ & $5(6.3)$ & $13(8.1)$ \\
\hline - Viudo & $2(2.5)$ & $0(0)$ & $2(1.3)$ \\
\hline \multicolumn{4}{|l|}{ Situación laboral [n (\%)] } \\
\hline - Activa & $18(22.5)$ & $58(72.5)$ & $76(47.5)$ \\
\hline - No activa & $62(77.5)$ & $22(27.5)$ & $84(52.5)$ \\
\hline \multicolumn{4}{|l|}{ Nivel educativo [n (\%)] } \\
\hline - Básico (primaria o menos) & $39(48.8)$ & $10(12.5)$ & $49(30.6)$ \\
\hline - Medio (Secundaria o FP) & $28(35)$ & $44(55)$ & $72(45)$ \\
\hline - Superior (Universitaria) & $13(16.3)$ & $26(32.5)$ & $39(24.4)$ \\
\hline
\end{tabular}

B) Análisis de la capacidad predictiva de las variables relevantes (psicológicas, sociodemográficas y del estrés percibido) sobre la sintomatología

Las características descriptivas (media y desviación típica) para cada grupo y para la muestra total, pueden verse en la Tabla 2. El contraste entre ambos grupos realizado mediante Análisis de Varianza, considerando como covariantes las variables sociodemográficas relevantes (sexo, edad, nivel educativo y situación laboral) mostró que el grupo clínico, frente al control, presentaba de forma estadísticamente significativa (todas las $\mathrm{F}$ con $p<.001)$ mayor neuroticismo $\left(\eta_{p}^{2}=.22\right)$, menor extraversión $\left(\eta_{p}^{2}=.05\right)$ y menor positividad $\left(\eta_{p}^{2}=.15\right)$, percibía mayor estrés $\left(\eta_{p}^{2}=.11\right)$, utilizaba en mayor medida

Tabla 2

Estadísticos descriptivos, media y desviación típica (entre paréntesis), de las variables de personalidad, afrontamiento, estrés percibido y sintomatología para cada grupo y para la muestra total

\begin{tabular}{lccc}
\hline & $\begin{array}{c}\text { Grupo clínico } \\
(N=80)\end{array}$ & $\begin{array}{c}\text { Grupo control } \\
(N=80)\end{array}$ & $\begin{array}{c}\text { Muestra total } \\
(N=160)\end{array}$ \\
\hline Extraversión & $24.46(6.02)$ & $27.46(5.04)$ & $25.96(5.73)$ \\
Neuroticismo & $27.60(5.80)$ & $19.01(6.83)$ & $23.31(7.65)$ \\
Positividad & $23.24(5.42)$ & $29.25(5.83)$ & $26.24(6.37)$ \\
Compromiso & $1.79(.68)$ & $2.02(.50)$ & $1.91(.61)$ \\
Apoyo & $1.86(.81)$ & $1.84(.79)$ & $1.85(.80)$ \\
Falta de compromiso & $1.08(.53)$ & $.68(.45)$ & $.88(.53)$ \\
Humor & $.62(.86)$ & $1.21(.93)$ & $.92(.94)$ \\
Religión & $.94(1.02)$ & $1.04(1.08)$ & $.99(1.05)$ \\
Estrés percibido & $1.68(.47)$ & $1.29(.46)$ & $1.48(.50)$ \\
Ansiedad & $25.64(10.60)$ & $6.98(7.01)$ & $15.66(14.78)$ \\
Depresión & $24.76(14.13)$ & $6.55(8.50)$ & $16.31(12.95)$ \\
\hline
\end{tabular}


el afrontamiento de falta de compromiso $\left(\eta_{p}^{2}=.10\right) \mathrm{y}$ en menor medida el de humor $\left(\eta_{p}^{2}=.07\right)$, e informaba de más síntomas de ansiedad $\left(\eta_{p}^{2}=.22\right)$ y de depresión $\left(\eta^{2} p\right.$ $=.36$ ).

Para analizar la sintomatología, se realizaron análisis de regresión lineal múltiple en primer lugar para la muestra total (ver Tabla 3), tomando como predictores las variables de personalidad medidas (extraversión, neuroticismo y positividad), los cinco grupos de estrategias de afrontamiento (compromiso, falta de compromiso, búsqueda de apoyo, humor y religión), las características sociodemográficas relevantes previamente señaladas (sexo, edad, nivel educativo y situación laboral), el estrés percibido y el grupo.

Tabla 3

Resultados del análisis de regresión de las variables sociodemográficas, estrés percibido, personalidad y afrontamiento sobre depresión y ansiedad en la muestra total $(N=160)$

\begin{tabular}{|c|c|c|c|c|}
\hline Criterio: Depresión & $\beta$ & $t$ & $F_{(14,145)}$ & $R^{2}$ ajustado \\
\hline Grupo & -.29 & $-4.42^{* * *}$ & $27.84^{* * *}$ & .70 \\
\hline Sexo & -.07 & -1.37 & & \\
\hline Edad & .04 & .84 & & \\
\hline Nivel educativo & -.08 & -1.43 & & \\
\hline Situación laboral & .18 & $3.43^{* * *}$ & & \\
\hline Estrés percibido & .13 & $2.37^{*}$ & & \\
\hline Extraversión & -.10 & $-2.02^{*}$ & & \\
\hline Neuroticismo & .16 & $2.26^{*}$ & & \\
\hline Positividad & -.21 & $-3.16^{* *}$ & & \\
\hline Afront. de compromiso & .07 & 1.27 & & \\
\hline Afront. de apoyo & -.04 & -.69 & & \\
\hline Afront. de falta de compromiso & .16 & $2.99^{\star *}$ & & \\
\hline Afront. de humor & -.03 & -.52 & & \\
\hline Afront. de religión & .03 & .52 & & \\
\hline Criterio: Ansiedad & $\beta$ & $t$ & $F_{(14,145)}$ & $R^{2}$ ajustado \\
\hline Grupo & -.22 & $-2.61^{* *}$ & $14.27^{* * *}$ & .54 \\
\hline Sexo & -.03 & -.53 & & \\
\hline Edad & .11 & $1.87^{\circ}$ & & \\
\hline Nivel educativo & -.13 & $-1.97^{*}$ & & \\
\hline Situación laboral & .18 & $2.79^{\star \star}$ & & \\
\hline Estrés percibido & .12 & $1.80^{\circ}$ & & \\
\hline Extraversión & -.07 & -1.15 & & \\
\hline Neuroticismo & .29 & $3.25^{\star \star \star}$ & & \\
\hline Positividad & -.09 & -1.06 & & \\
\hline Afront. de compromiso & .05 & .80 & & \\
\hline Afront. de apoyo & .12 & 1.61 & & \\
\hline Afront. de falta de compromiso & .06 & .88 & & \\
\hline Afront. de humor & .01 & .02 & & \\
\hline Afront. de religión & .09 & 1.38 & & \\
\hline
\end{tabular}

Nota. (1) Método: Introducir; (2) $\beta$ : Coeficiente de regresión estandarizado; (3) $t$ : prueba t de Student; (4) $R^{2}$ ajustado: Coeficiente de determinación ajustado; (5) ${ }^{\circ} p<.07$; ${ }^{*} p<.05$; ${ }^{* *} p<.01$; ${ }^{* *} p<.001$; (6) Situación laboral: $1=$ Activa, 2 = No activa; Grupo: 1 = Clínico, 2 = Control; Sexo: 1= Hombre, 2 = Mujer; (7) Afront.: Afrontamiento. 
Tabla 4

Correlaciones entre las variables psicológicas analizadas para el grupo clínico (por debajo de la diagonal) y control (por encima de la diagonal

\begin{tabular}{|c|c|c|c|c|c|c|c|c|c|c|c|}
\hline & 1 & 2 & 3 & 4 & 5 & 6 & 7 & 8 & 9 & 10 & 11 \\
\hline 1. Extraversión & 1 & -.24 & $.29^{\prime \prime}$ & .30 & .19 & -.21 & -.04 & -.17 & .13 & -.31 & -.17 \\
\hline 2. Neuroticismo & $-.42^{\pi \pi \pi}$ & 1 & $-.41^{\pi \pi \pi}$ & -.15 & -.01 & $.58^{\pi \times \pi}$ & -.14 & $.23^{\pi}$ & $.25^{\pi}$ & $.56^{\pi \approx \pi}$ & $.58^{\star \star \star}$ \\
\hline 3. Positividad & $.33^{\pi \pi}$ & $-.40^{\pi \pi \pi}$ & 1 & $.38^{\pi \times x}$ & $.51^{\pi \pi \pi}$ & -.13 & -.01 & $.23^{x}$ & -.18 & $-.48^{\pi \times \pi}$ & $-.32^{* *}$ \\
\hline 4. Af. Compromiso & .12 & $-.29^{\pi \pi}$ & $.39^{\pi \times \pi}$ & 1 & $.50^{\pi \times \pi}$ & -.03 & .13 & .19 & .06 & $-.26^{\pi}$ & $-.22^{*}$ \\
\hline 5. Af. Apoyo & .17 & -.14 & $.41^{\pi \pi \pi}$ & $.49^{\pi x \pi}$ & 1 & .08 & .22 & $.30^{x \times x}$ & .18 & -.21 & .01 \\
\hline 6. Af. Falta Compr. & .06 & .21 & -.08 & -.06 & .12 & 1 & -.07 & $.36^{\star * *}$ & .14 & $.42^{\star \star \star *}$ & $.43^{\star \star *}$ \\
\hline 7. Af. Humor & $.24^{\pi}$ & $-.26^{\pi}$ & .15 & $.47^{\pi \pi \pi}$ & $.23^{\pi}$ & -.04 & 1 & -.01 & .06 & -.20 & -.17 \\
\hline 8. Af. Religión & .05 & .19 & .15 & .08 & $.29^{\star *}$ & .18 & .11 & 1 & .10 & .10 & $.26^{*}$ \\
\hline 9. Estrés Percibido & .03 & $.28^{\star}$ & -.09 & .06 & -.03 & .09 & .09 & .16 & 1 & $.29^{\pi x}$ & $.29^{* *}$ \\
\hline 10. Depresión & $-.27^{\star}$ & $.38^{\pi \times \pi}$ & $-.40^{\pi \pi \pi}$ & -.09 & -.21 & $.27^{\pi}$ & -.03 & .09 & $.26^{\star}$ & 1 & $.69^{* * *}$ \\
\hline 11. Ansiedad & -.18 & $.31^{\pi \pi}$ & -.08 & .08 & .10 & .16 & .02 & $.25^{\star}$ & $.22^{\pi}$ & $.61^{\pi \pi x}$ & 1 \\
\hline
\end{tabular}

Nota. ${ }^{*} p<.05 ;{ }^{* *} p<.01 ;{ }^{* * *} p<.001$

\section{Tabla 5.}

Resultados del análisis de regresión de las variables sociodemográficas, estrés percibido, personalidad y afrontamiento sobre depresión y ansiedad en el grupo clínico $(N=80)$

\begin{tabular}{|c|c|c|c|c|}
\hline Criterio: Depresión & $\beta$ & $t$ & $F_{(9,70)}$ & $R^{2}$ ajustado \\
\hline Sexo & -.22 & $-2.21^{*}$ & $5.66^{* * *}$ & .34 \\
\hline Edad & .12 & 1.25 & & \\
\hline Nivel educativo & -.09 & -.96 & & \\
\hline Situación laboral & .23 & $2.40^{*}$ & & \\
\hline Estrés percibido & .15 & 1.49 & & \\
\hline Extraversión & -.17 & -1.58 & & \\
\hline Neuroticismo & .14 & 1.20 & & \\
\hline Positividad & -.34 & $-3.27^{* *}$ & & \\
\hline Afront. de falta de compromiso & .22 & $2.29^{*}$ & & \\
\hline Criterio: Ansiedad & $\beta$ & $t$ & $F_{(7,72)}$ & $R^{2}$ ajustado \\
\hline Sexo & -.13 & -1.23 & $3.23^{* *}$ & .17 \\
\hline Edad & .18 & 1.60 & & \\
\hline Nivel educativo & -.10 & -.96 & & \\
\hline Situación laboral & .14 & 1.35 & & \\
\hline Estrés percibido & .13 & 1.10 & & \\
\hline Neuroticismo & .30 & $2.65^{\star *}$ & & \\
\hline Afront. de religión & .14 & 1.27 & & \\
\hline
\end{tabular}

Nota. (1) Método: Introducir, tomando como predictores las variables sociodemográficas relevantes y las psicológicas significativamente correlacionadas con cada criterio; (2) $\beta$ : Coeficiente de regresión estandarizado (3) $t$ : prueba t de Student; (4) $R^{2}$ ajustado: Coeficiente de determinación ajustado; (5) ${ }^{*} p<.05 ;{ }^{* *} p<.01$; ${ }^{* * *} p<.001$; (6) Situación laboral: $1=$ Activa, $2=$ No activa; Sexo: 1 = Hombre, 2 = Mujer; (7) Afront.: Afrontamiento. 
En la muestra total se obtuvo un modelo significativo que explicaba el $70 \%$ de la varianza de la sintomatología depresiva $\left(\mathrm{F}_{(14,145)}=27.84, p<.001\right)$. Según el modelo, un mayor neuroticismo, menor extraversión y positividad, mayor afrontamiento de falta de compromiso, mayor estrés percibido, estar en situación laboral no activa y pertenecer al grupo clínico predijeron una mayor depresión (ver Tabla 3).

Al resultar significativa la variable grupo, se realizaron posteriormente los mismos análisis para cada uno por separado. No obstante, en estos análisis, al reducirse el tamaño de la muestra, sólo se incluyeron como predictores, además de las variables sociodemográficas, aquellas de personalidad, afrontamiento y percepción de estrés que correlacionaran significativamente con los síntomas de depresión (ver Tabla 4).

En el caso del grupo clínico (ver Tabla 5), la capacidad predictiva de las variables sobre la sintomatología depresiva fue del $34 \%\left(\mathrm{~F}_{(9,70)}=5.66, p<.001\right)$. Según el modelo, menor positividad, mayor afrontamiento de falta de compromiso, estar en situación laboral no activa y ser hombre predijeron una mayor depresión. En el caso del grupo control (ver Tabla 6), la varianza predicha fue del $50 \%\left(\mathrm{~F}_{(10,69)}=8.85, p<.001\right)$, resultando significativas en este caso el neuroticismo y la situación laboral no activa, y de forma marginalmente significativa, la baja positividad y el estrés percibido.

Tabla 6

Resultados del análisis de regresión de las variables sociodemográficas, estrés percibido, personalidad y afrontamiento sobre depresión y ansiedad en el grupo control $(N=80)$

\begin{tabular}{|c|c|c|c|c|}
\hline Criterio: Depresión & $\beta$ & $\bar{t}$ & $F_{(10,69)}$ & $R^{2}$ ajustado \\
\hline Sexo & .01 & .05 & $8.85^{\star \star \star}$ & .50 \\
\hline Edad & .03 & .33 & & \\
\hline Nivel educativo & -.12 & -1.17 & & \\
\hline Situación laboral & .28 & $3.20^{* *}$ & & \\
\hline Estrés percibido & .16 & 1.81 & & \\
\hline Extraversión & -.13 & -1.46 & & \\
\hline Neuroticismo & .32 & $2.68^{* *}$ & & \\
\hline Positividad & -.19 & $-1.91^{\circ}$ & & \\
\hline Afront. de compromiso & -.04 & -.42 & & \\
\hline Afront. de falta de compromiso & .18 & 1.65 & & \\
\hline Criterio: Ansiedad & $\beta$ & $t$ & $F_{(10,69)}$ & $R^{2}$ ajustado \\
\hline Sexo & .18 & $1.88^{\circ}$ & $7.98^{\star \star \star}$ & .47 \\
\hline Edad & .00 & .03 & & \\
\hline Nivel educativo & -.12 & -1.12 & & \\
\hline Situación laboral & .27 & $2.96^{\star \star}$ & & \\
\hline Estrés percibido & .11 & 1.25 & & \\
\hline Neuroticismo & .39 & $3.16^{\star *}$ & & \\
\hline Positividad & -.10 & -.97 & & \\
\hline Afront. de compromiso & -.14 & -1.47 & & \\
\hline Afront. de falta de compromiso & .10 & .88 & & \\
\hline Afront. de religión & 14 & 1.46 & & \\
\hline
\end{tabular}

Notas. (1) Método: Introducir, tomando como predictores las variables sociodemográficas relevantes y las psicológicas significativamente correlacionadas con cada criterio; (2) $\beta$ : Coeficiente de regresión estandarizado (3) $t$ : prueba $t$ de Student; (4) $R^{2}$ ajustado: Coeficiente de determinación ajustado; $(5){ }^{\circ} p<.07 ;{ }^{*} p<.05 ;{ }^{* *} p<.01 ;{ }^{* *} p<.001$; (6) Situación laboral: 1 = Activa, 2 = No activa; Sexo: 1 = Hombre, 2 = Mujer; (7) Afront.: Afrontamiento. 
En el caso de la capacidad predictiva de las variables sobre la sintomatología ansiosa en la muestra total, la ecuación predijo el $54 \%$ de la varianza de la ansiedad. Según el modelo obtenido $\left(\mathrm{F}_{(14,145)}=14.27, p<.001\right)$, un mayor neuroticismo, estar en situación laboral no activa, un nivel educativo más bajo y pertenecer al grupo clínico predijeron una mayor puntuación en ansiedad. También fueron predictores marginalmente significativos el aumento en la edad y un mayor estrés percibido (ver Tabla 3$)$.

En este caso también resultó significativa la variable grupo, realizándose los análisis igualmente para cada uno por separado, siguiendo el mismo criterio que en el caso de la depresión, es decir, tomando como predictores las variables de personalidad, afrontamiento y estrés que correlacionaran significativamente con los síntomas de ansiedad. En el caso del grupo clínico (ver Tabla 5), la capacidad predictiva de las variables sobre la sintomatología ansiosa fue del $17 \%\left(\mathrm{~F}_{(7,72)}=3.23, p<.01\right)$, resultando el neuroticismo el único predictor significativo. En el caso del grupo control (ver Tabla 6), la varianza predicha fue del $47 \%\left(\mathrm{~F}_{(10,69)}=7.98, p<.001\right)$, resultando significativas las variables de neuroticismo, situación laboral y de forma marginalmente significativa, el sexo, a favor de mayor sintomatología ansiosa en las mujeres.

\section{Discusión}

Los objetivos principales de este estudio fueron comprobar la capacidad predictiva de algunas variables psicológicas y sociodemográficas significativas sobre el nivel de sintomatología depresiva y ansiosa en una muestra de personas con Trastorno Adaptativo, una categoría diagnóstica muy utilizada, controvertida, y poco investigada, así como la comparación de los resultados con una muestra control.

En general, se encontró que, considerando la muestra total, la ansiedad y la depresión podían predecirse en un $54 \%$ y un $70 \%$, respectivamente, por las variables incluidas (ver Tabla 3), aunque los resultados variaban considerablemente al realizar por separado los análisis para cada grupo (clínico y control), reduciéndose el número de variables predictoras y la varianza explicada por éstas (ver Tablas 5 y 6). Estos resultados pueden deberse, entre otros factores, al menor tamaño de la muestra al considerar cada grupo por separado, al menor número de predictores significativos incluidos en las ecuaciones finales (por ejemplo, en la ecuación sobre la ansiedad, en el grupo clínico sólo resultó significativo el neuroticismo, siendo la capacidad predictiva de la ecuación la más baja, del $17 \%$ ), y especialmente a la consideración conjunta y potenciada de las interrelaciones entre los predictores en la muestra total, siendo relevantes diferentes variables cuando se realizan los análisis por separado para cada grupo. A continuación, se describen más exhaustivamente los resultados en base a los objetivos e hipótesis planteadas.

El primer objetivo fue estudiar las relaciones de la personalidad con los síntomas, planteándose como primera hipótesis que el neuroticismo predeciría positivamente tanto la sintomatología ansiosa como depresiva, mientras que mayor extraversión y positividad predecirían menor nivel de sintomatología. Esta hipótesis se cumplió al considerar la muestra total, pero sólo parcialmente considerando cada grupo por separado, aunque los resultados fueron en la misma línea, no siendo significativas las betas correspondientes. El neuroticismo resultó ser un predictor significativo de ambos tipos de síntomas únicamente en el grupo control, resultando sólo relevante en la predicción de la ansiedad en el grupo con TA (aunque en el caso de la depresión, los resultados fueron en la línea de los demás resultados, con una beta tipificada de .14 que no fue suficiente, sin embargo, para resultar significativa). La positividad predijo menos síntomas depresivos, pero no ansiosos, en ambos grupos. Y la extraversión sólo se relacionó con los síntomas depresivos en la muestra total, aunque al considerar cada grupo por separado las betas, aun siendo mayores y en igual dirección que con la muestra completa, no resultaron significativas, influyendo, posiblemente, el tamaño grupal y las interrelaciones entre los diferentes predictores considerados. Por tanto, tampoco se cumplió la quinta de las hipótesis relacionada con las diferencias entre grupos, dado que no resultaron predictivas las mismas variables (excepto en el caso de la positividad, donde los resultados fueron consistentes en ambos grupos). Estos resultados no resultarían compatibles con estudios como el metaanálisis de Malouff y colaboradores (2005), que 
encontraron que mayor neuroticismo y menor extraversión se relacionaría con más síntomas y trastornos tanto ansiosos como depresivos, $\mathrm{u}$ otros trabajos que relacionan el alto neuroticismo con ambos síntomas, y la baja extraversión sólo con la depresión (Kotov et al., 2010; Watson y Clark, 1992). Es probable que estos resultados se relacionen en parte con la diferenciación de la muestra de estudio en un grupo clínico y otro control, lo que supone al fin y al cabo dividir a los sujetos en altos y bajos en algunas características. Como consecuencia se ve afectada la dimensionalidad o linealidad de las mismas características, lo que afecta a las relaciones entre las variables. Por ejemplo, en el caso del grupo clínico, con más alto neuroticismo y mayor homogeneidad de la muestra al compartir un mismo diagnóstico que en el caso del grupo control, esta variable no predijo la depresión, a pesar de que es considerada uno de los predictores más relevantes de psicopatología ansiosa y depresiva. Aunque, como ya se ha comentado, parte de los resultados podrían explicarse por el menor tamaño de las submuestras.

Con respecto a la positividad, los resultados son acordes a la literatura, que la ha relacionado negativamente con el afecto negativo y la depresión (Caprara et al., 2012), lo cual es esperable considerando que es conceptualizada como un factor disposicional, relacionado con la tendencia a ver la vida y las experiencias desde un punto de vista positivo, con componentes tanto cognitivos-evaluativos como emocionales-afectivos que afectan a la interpretación de la situación y los propios recursos.

La segunda hipótesis, según la cual se esperaba encontrar que el uso de estrategias de afrontamiento de falta de compromiso predijese mayores niveles de sintomatología, y el resto predijese menores niveles, sólo se cumplió en su primera parte. Es decir, en nuestro estudio encontramos que un mayor uso del afrontamiento de falta de compromiso se relacionó con un aumento de la sintomatología depresiva, pero no ansiosa, en la muestra total y clínica, no en la control (y por tanto, en este caso, también se encontraron diferencias entre los grupos, aunque como en el caso de la personalidad, los resultados fueron en la misma línea, con una beta tipificada de .18 en el grupo control, superior incluso a la obtenida en la muestra total, pero insuficiente para resultar significa- tiva). Este resultado no resulta compatible con las relaciones encontradas tanto con depresión como con ansiedad (Carver y Connor-Smith, 2010), aunque podría explicarse por la relación encontrada en la literatura entre el afrontamiento más pasivo o evitativo y aquellas situaciones estresantes en que la persona valora que tiene escaso control percibido o autoeficacia, variables que a su vez guardan estrecha relación con la depresión (Bandura, 1997). Podría también sugerirse, que el mayor peso en el grupo clínico del afrontamiento de falta de compromiso o evitativo frente a un rasgo más estable como el neuroticismo en la predicción de la sintomatología depresiva, estaría indicando un efecto significativo de mediación, que podría no estar actuando en el grupo control, donde la sintomatología no es tan elevada ni alcanza niveles clínicos, siendo en este caso el peso predictivo del rasgo de neuroticismo mayor y de efecto más directo, hipótesis que debería abordarse en futuras investigaciones.

El resto de estrategias de afrontamiento (de compromiso, humor, búsqueda de apoyo social, y religión), consideradas generalmente como más activas y dirigidas al manejo del problema o de las emociones asociadas, no se asoció a una disminución de síntomas, tal como se esperaba. No obstante, en la revisión de Taylor y Stanton (2007) se concluyó que, aunque en general el afrontamiento de aproximación o de compromiso se relaciona positivamente con la salud física y psicológica en circunstancias estresantes, los resultados son menos concluyentes que los encontrados con el afrontamiento evitativo o de falta de compromiso. Esto puede ser debido a diversos factores como, por ejemplo, el hecho de que algunas estrategias como la solución de problemas, aunque pueden promover el afecto positivo, no resultan útiles para el manejo de los aspectos inmutables o incontrolables del estresor, repercutiendo en la eficacia del afrontamiento y en la salud mental general.

En cuanto a la tercera hipótesis, relacionada con la capacidad predictiva del estrés percibido, se esperaba que predijese mayores niveles de síntomas tanto ansiosos como depresivos, pero también en este caso los resultados sólo se cumplieron en parte. Se encontró que mayor estrés percibido predijo mayor depresión considerando la muestra total, siendo casi significativo en la predicción de la ansiedad. No obstante, no resultó un predictor signifi- 
cativo considerando los grupos por separado (fue casi significativo en la predicción de la depresión en el grupo clínico, pero no lo fue en el control, aunque como en el afrontamiento y la personalidad, también en este caso los resultados fueron en la misma línea aunque sin alcanzar la significación estadística). Por tanto, en este caso los resultados sí que fueron similares en ambos grupos. Es probable que debido a la estrecha relación del estrés percibido con otras variables de estudio, como la personalidad o el afrontamiento, al considerarse conjuntamente con ellas pierda significación en la predicción de los síntomas, lo cual debería ser analizado en posteriores estudios.

Por último, con respecto a la cuarta hipótesis, relativa a las variables sociodemográficas, tampoco se encontraron los resultados esperados, aunque en este caso no hubo muchas diferencias entre los grupos. Únicamente resultó significativa la situación laboral, que predijo la sintomatología tanto ansiosa como depresiva, independientemente de la muestra considerada, lo cual resulta compatible en general con los hallazgos previos (Altemus et al., 2014; Tang et al., 2014), y coherente si se considera que el hecho de no tener trabajo puede ser en sí mismo una situación estresante que dé lugar a sintomatología. Los resultados en las demás variables sociodemográficas fueron más inconsistentes (menor nivel educativo predijo mayor ansiedad en la muestra total, siendo casi significativa la edad, y ser hombre predijo más depresión sólo en la clínica). No obstante, la literatura ha encontrado que las mujeres, determinados grupos de edad y las personas con menor nivel educativo tienen mayores niveles de psicopatología, lo que se ha asociado en muchos casos a las diferencias en roles sociales y recursos disponibles (Altemus et al., 2014; Tang et al., 2014). Por tanto, el resultado debería ser también estudiado más en profundidad, ya que podría haber influido igualmente el hecho de considerar en los mismos análisis otras variables de mayor relevancia.

En resumen, en este estudio se encontró que la positividad predecía mayores puntuaciones en depresión independientemente de la muestra; el neuroticismo predecía también mayor ansiedad en ambas muestras, pero sólo era relevante en la predicción de la depresión en el grupo control; el afrontamiento sólo era relevante en el grupo clínico, donde mayor uso del afrontamiento de falta de compromiso predecía más síntomas depresivos, mientras que el estrés percibido mostraba valores cercanos a la significación en la predicción de síntomas depresivos en el grupo control; y por último, la variable sociodemográfica más consistentemente asociada a los síntomas fue estar en paro, prediciendo tanto síntomas ansiosos como depresivos. Por tanto, en base a los resultados de este trabajo puede afirmarse que tanto la personalidad como el afrontamiento, junto con algunas características sociodemográficas y el estrés percibido por la persona, podrían considerarse factores de vulnerabilidad que pueden influir en el desarrollo y gravedad de la sintomatología ansiosa y depresiva (tanto en muestras generales como clínicas, en este caso con TA), aunque con diferente peso según la muestra considerada.

Las aplicaciones clínicas son evidentes. Por un lado, con respecto a la evaluación clínica, a partir de varios autoinformes breves, de personalidad, afrontamiento y estrés percibido, se podría predecir el nivel de sintomatología ansiosa y depresiva en sujetos sometidos a estrés vital, sin psicopatología y con TA. Y por otro lado, con respecto al abordaje psicoterapéutico, los resultados apuntarían a la recomendación de intervenciones preventivas o terapéuticas, en muestras generales y clínicas respectivamente, dirigidas al desarrollo de estrategias, en primer lugar, de autorregulación emocional, para disminuir la tendencia a experimentar emociones negativas, y así prevenir el desarrollo o empeoramiento de síntomas tanto ansiosos como depresivos (especialmente en la población general); en segundo lugar, de cara a un mejor afrontamiento del estrés vital (minimizando el uso de estrategias pasivas o evitativas), lo que repercutiría en un menor nivel de síntomas depresivos en los sujetos con TA; y por último, dirigidas a la modificación de los procesos cognitivos o valorativos (sobre la situación o sobre los propios recursos), lo que influiría en una disminución de los síntomas depresivos tanto en muestras generales como con TA.

A pesar de lo anterior, el estudio realizado tiene algunas limitaciones, relacionadas principalmente con la metodología. En primer lugar, se trata de un estudio correlacional por lo que no pueden establecerse relaciones de causalidad. En segundo lugar, sólo se usan autoinfor- 
mes retrospectivos, pudiendo verse afectados los resultados por sesgos de memoria o por el estado afectivo de los sujetos (Caprara et al., 2010; Gärling y Gamble, 2012). Con respecto a los análisis realizados, no se estudia el papel moderador de las variables de personalidad y afrontamiento entre el estrés y la sintomatología, ni el papel mediador del estrés y el afrontamiento entre la personalidad y la sintomatología, ya que excedía los objetivos de este trabajo, además de la necesidad de muestras más amplias, aunque sería interesante para una futura investigación. Y, por último, en este estudio no se consideran variables muy relevantes, por ejemplo, relacionadas con el estrés experimentado, que tienen que ver con aspectos valorativos como la controlabilidad y la predictibilidad, y que guardan gran relación con el estrés percibido y con las respuestas que el sujeto pone en marcha (Sandín, 2008).

\section{Referencias}

Alessandri, G., Caprara, G. V. y Tisak, J. (2012). The unique contribution of positive orientation to optimal functioning: Further explorations. European Psychologist, 17, 44-54. http://dx.doi.org/ 10.1027/1016-9040/a000070

Altemus, M., Sarvaiya, N. y Epperson, C. N. (2014). Sex differences in anxiety and depression clinical perspectives. Frontiers in Neuroendocrinology, 35, 320-330.

http://dx.doi.org/10.1016/j.yfrne.2014.05.004

American Psychiatric Association (APA). (2000). Diagnostic and Statistical Manual of Mental Disorders, 4th Ed, Text Revised (DSM-IV-TR). Washington, D.C.: APA.

American Psychiatric Association (APA). (2013). Diagnostic and Statistical Manual of Mental Disorders, 5th Ed. (DSM-V). Arlington, VA: APA. http://dx.doi.org/10.1176/appi.books.978089042559 6
Bandura, A. (1997). Self-efficacy: The exercise of control. New York: Freeman.

Beck, A. T., Brown, G., Epstein, N. y Steer, R. A. (1988). An Inventory for Measuring Clinical Anxiety: Psychometric properties. Journal of Consulting and Clinical Psychology, 56, 893-897. http://dx.doi.org/10.1037/0022-006X.56.6.893

Beck, A. T., Rush, A.J., Shaw, B. y Emery, G. (1979). Cognitive therapy of depression. Nueva York: Guilford Press.

Beck, A. T., Steer, R. A. y Garbin, M. G. (1988) Psychometric properties of the Beck Depression Inventory: Twenty-five years of evaluation. Clinical Psychology Review, 8, 77-100. http://dx.doi.org/10.1016/0272-7358(88)90050-5

Benet-Martínez, V. y John, O. P. (1998). Los Cinco Grandes across cultures and ethnic groups: Multitrait multimethod analyses of the Big Five in Spanish and English. Journal of Personality and Social Psychology, 75, 729-750. http://dx.doi.org/10.1037/0022-3514.75.3.729

Bermúdez, M. P., Teva, I. y Buela-Casal, G. (2009). Influencia de variables sociodemográficas sobre los estilos de afrontamiento, el estrés social y la búsqueda de sensaciones sexuales en adolescentes. Psicothema, 21, 220-226. http://dx.doi.org/10.6018/analesps.31.1.163681

Caprara, G. V., Alessandri, G., Eisenberg, N., Kupfer, A., Steca, P., ... y Abela, J. (2012). The Positivity Scale. Psychological Assessment, 24, 701-712. http://dx.doi.org/10.1037/a0026681

Caprara, G. V., Steca, P., Alessandri, G., Abela, J. R. Z. y McWhinnie, C. M. (2010). Positive orientation: Explorations of what is common to life satisfaction, self-esteem, and optimism. Epidemiology and Psychiatric Sciences, 19, 63-71. http://dx.doi.org/ 10.1017/S1121189X00001615 
Carver, C. S. (1997). You want to measure coping but your protocol's too long: Consider the Brief COPE. International Journal of Behavioral Medicine, 4, 92-100.

http://dx.doi.org/10.1207/s15327558ijbm0401_6

Carver, C.S. y Connor-Smith, J. (2010). Personality and coping. Annual Review of Psychology, 61, 679-704. http://dx.doi.org/10.1146/annurev.psych.093008.10 0352

Casey, P., Dowrick, C. y Wilkinson, G. (2001). Adjustment disorders: Fault line in the psychiatric glossary. British Journal of Psychiatry, 179, 479481. http://dx.doi.org/10.1192/bjp.179.6.479

Ferrando, L., Franco, A. L., Soto, M., Bobes, J., Soto, O., Franco, L. y Gibert, J. (1998). MINI Entrevista Neuropsiquiátrica Internacional (Versión en español 5.0.0.) DSM-IV. Madrid, España: Instituto IAP.

For-Wey, L., Fei-Yin, L. y Bih-Ching, S. (2002). The relationship between life adjustment and parental bonding in military personnel with adjustment disorder in Taiwan. Military Medicine, 167, 678682 .

Gärling, T., y Gamble, A. (2012). Influences on current mood of eliciting life-satisfaction judgments. The Journal of Positive Psychology, 7, 219-229. http://dx.doi.org/10.1080/17439760.2012.674547

IBM Corp (2010). IBM SPSS. Statistics for Windows, Version 19.0. Armonk, NY: IBM Corp.

Kotov, R., Gamez, W., Schmidt, F. y Watson, D. (2010). Linking "Big', personality traits to anxiety, depressive, and substance use disorders: A metaanalysis. Psychological Bulletin, 136, 768-821. http://dx.doi.org/10.1037/a0020327

Lahey, B. B. (2009). Public health significance of neuroticism. American Psychologist, 64, 241-256. http://dx.doi.org/10.1037/a0015309
Li, R., Cooper, C., Bradley, J., Shulman, A. y Livingston, G. (2012). Coping strategies and psychological morbidity in family careers of people with dementia: A systematic review and meta-analysis. Journal of Affective Disorders, 139, 1-11. http://dx.doi.org/10.1016/j.jad.2011.05.055

López-Ibor, J. J., Pérez-Urdániz, A. y Rubio, V. (1996). Examen Internacional de los Trastornos de la Personalidad (IPDE): Módulo DSM-IV y CIE-10. Madrid, España: Meditor.

Loranger, A. W. (1995). International Personality Disorder Examination (IPDE). Ginebra, Suiza: Organización Mundial de la Salud. http://dx.doi.org/10.1001/archpsyc.1994.039500300 51005

Lucas, R. E. y Fujita, F. (2000). Factors influencing the relation between extraversion and pleasant affect. Journal of Personality and Social Psychology, 79, 1039-1056. http://dx.doi.org/10.1037/00223514.79.6.1039

Malouff, J. M., Thorsteinsson, E. B. y Schutte, N. S. (2005). The relationship between the five-factor model of personality and symptoms of clinical disorders: A meta-analysis. Journal of Psychopathology and Behavioral Assesment, 27, 101-114. http://dx.doi.org/10.1007/s10862-0055384-y

Mandelli, L., Nearchou, F.A., Vaiopoulos, C., Stefanis, C.N., Vitoratou, S., Serretti, ... y Stefanis, N.C. (2015). Neuroticism, social network, stressful life events: Association with mood disorders, depressive symptoms and suicidal ideation in a community sample of women. Psychiatry Research, 226, 38-44. http://dx.doi.org/10.1016/j.psychres.2014.11.001

McCrae, R. R. y Costa, P. T. (1991). Adding liebe und arbeit: The full five-factor model and well-being. Personality and Social Psychology Bulletin, 17, 227-232. http://dx.doi.org/10.1177/014616729101700217 
Mirnics, Z., Heincz, O., Bagdy, G., Surányi, Z., Gonda, X., ... y Juhasz, G. (2013). The relationship between the big five personality dimensions and acute psychopathology: mediating and moderating effects of coping strategies. Psychiatria Danuina, 25, 37988.

Morán, C., Landero, R. y González, M. T. (2010). COPE-28: un análisis psicométrico de la versión en español del Brief COPE [COPE-28: A psychometric analysis of the Spanish version of the Brief COPE]. Universitas Psychologica, 9, 543552.

Naragon-Gainey, K., Rutter, L. A. y Brown, T. A. (2014). The interaction of extraversion and anxiety sensitivity on social anxiety: evidence of specificity relative to depression. Behavioral Therapy, 45, 418429. http://dx.doi.org/10.1016/j.beth.2014.01.004.

Rammstedt, B. y John, O. P (2007). Measuring personality in one minute or less. A 10-item short version of the Big Five Inventory in English and German. Journal of Research in Personality, 41, 203-212.

http://dx.doi.org/10.1016/j.jrp.2006.02.001

Sandin, B. (2008). El estrés [Stress]. En A. Belloch, B. Sandín y F. Ramos (Eds.), Manual de psicopatología (Vol. II, pp. 3-42). Madrid, España: McGraw-Hill.

Sanz, J., García-Vera, M. P. y Fortún, M. (2012). El Inventario de Ansiedad de Beck (BAI): propiedades psicométricas de la versión española en pacientes con trastornos psicológicos [The Beck Anxiety Inventory (BAI): Psychometric properties of the Spanish version in patients with psychological disorders]. Behavioral Psychology-Psicología Conductual, 20, 563-583.

Sanz, J. y Navarro, M. E. (2003). Propiedades psicométricas de una versión española del Inventario de Ansiedad de Beck (BAI) en estudiantes universitarios [Psychometric properties of a Spanish version of the Beck Anxiety Inventory
(BAI) in university students]. Ansiedad y Estrés, 9, 59-84.

Seiffge-Krenke, L. y Klessinger, N. (2000). Long-term effects of avoidant coping on adolescents' depressive symptoms. Journal of Youth and Adolescence, 29, 617-630.

Sheehan, D. V., Lecrubier, Y., Sheehan, K. H., Amorim, P., Janavs, J...., Dunbar, G. C. (1998). The MiniInternational Neuropsychiatric Interview (M.I.N.I.): The development and validation of a structured diagnostic psychiatric interview for DSM-IV and ICD-10. Journal of Clinical Psychiatry, 59, 22-33.

Tang, B., Liu, X., Liu, Y., Xue, C. y Zhang, L. (2014). A meta-analysis of risk factors for depression in adults and children after natural disasters. BMC Public Health, 14, 623. http://dx.doi.org/10.1186/14712458-14-623.

Taylor, S. E. y Stanton, A. L. (2007). Coping resources, coping processes, and mental health. Annual Review of Clinical Psychology, 3, 377-401. http://dx.doi.org/10.1146/annurev-clinpsy-032511143108

Vallejo-Sánchez, B. y Pérez-García, A. M. (2015a). Positividad y afrontamiento en pacientes con trastorno adaptativo [Positivity and coping in patients with adjustment disorder]. Anales de Psicología, 31, 462-471. http://dx.doi.org/10.6018/analesps.31.2.176631

Vallejo-Sánchez, B. y Pérez-García, A.M. (2015b). The role of personality and coping in adjustment disorder. Clinical Psychologist, 19(1). http://dx.doi.org/10.1111/cp.12064

Vázquez, C. y Sanz, J. (1997). Fiabilidad y valores normativos de la versión española del Inventario para la Depresión de Beck de 1978 [Reliability and normative values of the Spanish version of the Beck Depression Inventory of 1978]. Clínica y Salud, 8, 403-422. 
Visser, M. M., Heijenbrok-Kal, M. H., Spijker, A. V., Oostra, K. M., Busschbach, J. J. y Ribbers, G. M. (2015). Coping, problem solving, depression, and health-related quality of life in patients receiving

Watson, D. y Clark, L. A. (1992). On traits and temperament: General and specific factors of emotional experience and their relation to the fivefactor model. Journal of Personality, 60, 441-476. http://dx.doi.org/10.1111/j.1467-

6494.1992.tb00980.x outpatient stroke rehabilitation. Archives of Physical Medicine and Rehabilitation, 96, 1492-1498. http://dx.doi.org/10.1016/j.apmr.2015.04.007

World Health Organization (WHO). (1992). International classification of diseases and related health problems, 10th revision. Ginebra, Suiza: WHO.

\title{
LIFE STRESS: PSYCHOLOGICAL AND SOCIO-DEMOGRAPHIC VARIABLES AS PREDICTORS OF EMOTIONAL DISTRESS
}

\author{
BEATRIZ VALLEJO-SÁNCHEZ Y ANA M. PÉREZ-GARCÍA
}

\section{EXTENDED SUMMARY}

\section{Introduction}

During lifetime, one has to face multiple stressful situations to which we can either, adapt to satisfactorily, or develop symptoms that may call for help or treatment at a given time. Personality and coping used to manage stress play an important role in the development of mental health problems. Specifically, high neuroticism, low positivity and disengagement coping prevent an adequate reaction to the problem itself. Furthermore, they have been associated with the presence of psychopathology (Li, Cooper, Bradley, Shulman, \& Livingston, 2012; Malouff, Thorsteinsson, \& Schutte, 2005). However, very few previous studies specifically examine personality and coping in people with "Adjustment Disorder" (AD) or "reaction to stress". A frequent diagnosis, as described in the classifications DSM-V (APA, 2013) and CIE-10 (WHO, 1992), is a maladaptive reaction to identifiable psychosocial stressors that comprise a wide variety of emotional symptoms or behaviour, as well as a significant deterioration in social or occupational functioning. Some studies have found that these variables are also relevant in samples with AD (For-Wey, Fei-Yin, \& Bih-Ching, 2002; Vallejo-Sánchez \& Pérez-García, $2015 \mathrm{a}, 2015 \mathrm{~b})$. Therefore, it would be interesting to carry out comparative studies with general samples or with other mental disorders, to analyse if personality and coping have different weight depending on the group, and contribute to the development of evaluation and intervention strategies.

The aim of this study was to analyse whether these psychological variables, as well as perceived stress and some relevant socio-demographic characteristics, could predict the presence and severity of anxious and depressive symptoms in a sample of people with $\mathrm{AD}$, compared to a control group.

\section{Method}

\section{Participants}

This cross-sectional study was carried out on 160 subjects divided into two groups: patients diagnosed with AD (WHO, 1992; APA, 2013) and a control group. 
The clinical group was made up of 80 outpatients who had been seen at the Mental Health Unit Santa Barbara Hospital and were diagnosed with $\mathrm{AD}$ through an initial, non-structured clinical interview. The presence of other psychopathology was discharged through two structured interviews: The International Mini Neuropsychiatric Interview (Sheehan et al., 1998; Spanish version by Ferrando et al., 1998) and the International Personality Disorders Examination (Loranger, 1995; Spanish version by López-Ibor, Pérez, \& Rubio, 1996).

The control group consisted of 80 people that had experienced at least one major stressful life event, but without mental health problems.

\section{Measures and procedure}

To measure perceived stress, a Life Events Scale was made, which consisted in a list of 40 stressful events to assess the occurrence and associated stress.

To measure personality, the Big-Five Inventory-44 (BFI-44; Benet-Martínez \& John, 1998) was used. It is a self-administered inventory comprised of 44 items, which assess the dimensions of normal personality described according to the Big-Five model (Costa \& McCrae, 1992): Extraversion, Agreeableness, Conscientiousness, Neuroticism, and Openness. Only Extraversion $(\alpha=.70)$ and Neuroticism $(\alpha=.84)$ were applied in this study.

To measure positive orientation, the Positivity Scale (Caprara, 2011; Spanish version by Caprara et al., 2012) was used. It is a brief self-assessed scale consisting of 8 items which assessed the positivity or propensity to think positively about oneself, life and the future $(\alpha=.83)$.

To measure coping, the Brief COPE (Carver, 1997; Spanish version by Morán, Landero, \& González, 2010) was used. It is a self-assessed inventory, initially organised into 28 items and 14 scales (two items in each scale). In this study, to maximise the reliability of the scales, the strategies were grouped into five categories following previous results (Vallejo-Sánchez \& PérezGarcía, 2015a): Humour coping $(\alpha=.83)$, religion coping $(\alpha=.84)$, engagement coping $(\alpha=.81)$, disengagement coping $(\alpha=.89)$ and social support coping $(\alpha=.88)$.

Finally, depressive symptoms were evaluated through the Beck Depression Inventory (Beck, Rush, Shaw, \& Emery, 1979; Spanish version by Vázquez \& Sanz, 1997), a 21 items self-assessed inventory $(\alpha=.94)$; and anxious symptoms were assessed through the Beck Anxiety Inventory (Beck, Brown, Epstein, \& Steer, 1988; Spanish version by Sanz \& Navarro, 2003), a selfinventory of 21 items $(\alpha=.96)$.

All the subjects meeting the inclusion criteria requirements were assessed through a protocol approved by the Hospital's Ethics Committee, which included a sheet of socio-demographic data and the previously described instruments completed at home. All the subjects who agreed to participate signed a consent form.

\section{Statistical analysis}

Statistical analyses were conducted using SPSS for Windows (version 19.0; IBM Corp., Armonk, NY, USA). To determine whether there were differences between the groups (clinical and control), $\chi^{2}$ tests (in the categorical variables) and analysis of variance (in the continuous variables) were used. Multiple regression analyses were carried out to predict symptomatology considering personality, coping, perceived stress and sociodemographic factors as predictors.

\section{Results}

There were no significant differences in the distribution of sex $\left(\chi^{2}=1.34, \mathrm{gl}=1, p=.16\right)$ and marital status $\left(\chi^{2}=3.98, \mathrm{gl}=3, p=.26\right)$. However, there were differences found in employment status $\left(\chi^{2}=40.10\right.$, $\mathrm{gl}=1, p<.001)$, educational level $\left(\chi^{2}=25.05, \mathrm{gl}=2\right.$, $p<.001$ ) and age $\left[\mathrm{F}_{(1,158)}=3.49, p=.06\right]$ (almost significant). The socio-demographic variables that showed differences between the clinical group and the control 
group were considered in subsequent statistical analyses. Although the number of women and men was equivalent in both groups, this variable was also considered because it is always advisable to control its influence on symptomatology.

These relevant socio-demographic variables (educational level, employment status, age and gender) were controlled in the MANOVA of a factor (clinical and control group) carried out. The clinical group reported (all F's with $p<.001$ ) greater neuroticism $\left(\eta^{2}=.22\right)$ and less extraversion $\left(\eta^{2} p=.05\right)$ and positivity $\left(\eta^{2}{ }^{2}=.15\right)$ than the control group. They also used disengagement strategies more $\left(\eta^{2}=.10\right)$ and humour strategies less $\left(\eta^{2} p=.07\right)$. Finally, the clinical group perceived higher levels of stress $\left(\eta^{2} p=.11\right)$, and reported more symptoms of anxiety $\left(\eta^{2} p=.22\right)$ and depression $\left(\eta^{2} p=.36\right)$ than the control group.

To test the predictive capacity of personality factors (extraversion, neuroticism, and positivity), coping strategies (humour, religion, engagement, disengagement and social support), perceived stress and socio-demographic variables (sex, age, educational level and employment status) on symptomatology, regression analyses were carried out.

In the total sample, a significant model was obtained that explained $70 \%$ of depressive symptomatology $\left(\mathrm{F}_{(14,145)}=27.84, p<.001\right)$. The significant variables were: more neuroticism, disengagement coping and perceived stress; less extraversion and positivity; as well as being unemployed and belonging to the clinical group.

The same analyses were made for each group separately, since this variable was a significant predictor of both anxious and depressive symptoms. In the clinical group, the significant model obtained $\left(\mathrm{F}_{(9,70)}=5.66\right.$, $p<.001$ ) accounted for $34 \%$ of the depressive symptoms. Lower positivity, greater use of disengagement or avoidant coping, being a male and unemployment predicted higher depressive symptomatology. Whereas in the control group, the significant model obtained $\left(\mathrm{F}_{(10,69)}=8.85, p<.001\right)$ accounted for $50 \%$ of these symptoms. In this case, more neuroticism and being unemployed result in higher depressive symptoms.
In the case of anxiety, the predictive capacity of the regression model was $54 \%\left(\mathrm{~F}_{(14,145)}=14.27, p<.001\right)$. Further anxious symptoms were predicted by: greater neuroticism, being unemployed, lower educational level and belonging to the clinical group. Regarding the clinical group, the predictive capacity of variables by anxious symptoms was only $17 \%\left(\mathrm{~F}_{(7,72)}=3.23, p<.01\right)$, being neuroticism the only significant predictor. Whereas in the control group, the predicted variance was $47 \%$ $\left(\mathrm{F}_{(10,69)}=7.98, p<.001\right)$. Higher neuroticism, being a female, and unemployment predicted higher anxiety in this group.

\section{Discussion}

The aim of this study was to analyse the influence of personality, coping, perceived stress and some relevant socio-demographic variables on the symptoms of anxiety and depression in a sample of patients with $\mathrm{AD}$, as well as analysing if the significant predictor variables were the same in a control group.

The regression analysis results, considering the total sample, indicated that anxiety could be predicted on a $54 \%$ by higher scores in neuroticism, less educational level, being unemployed and belonging to the clinical group. Whereas in the case of depression, the predictive capacity of the regression model was even greater, $70 \%$, resulting in greater neuroticism, disengagement coping and perceived stress; less extraversion and positivity; as well as being unemployed and belonging to the clinical group as relevant predictors.

When analyses were performed separately for each group (clinical and control), the number of predictors and the variance decreased significantly, especially in the clinical group. Summarising: In the first place, less positivity predicted higher scores on depression regardless of the considered sample. Secondly, greater neuroticism also predicted greater anxiety in both samples, but it was only relevant in predicting depression in the control group. Thirdly, use of disengagement coping predicted 
higher scores on depression only, being only relevant in the clinical group. Fourthly, perceived stress predicted depressive symptoms in the control group, although this result was marginally significant. Finally, the sociodemographic variable most consistently associated with symptoms was being unemployed, predicting both anxious and depressive symptoms, whereas the educational level predicted greater anxiety only in the total sample, and sex -being male- predicted greater depression in the clinical group.

The above results relating psychological variables (personality, coping and perceived stress) to psychopathology are consistent with previous literature (Alessandri, Caprara, \& Tisak, 2012; Caprara et al., 2012; Carver \& Connor-Smith, 2010; Malouff et al., 2005; Visser et al., 2015). The influence of socio-demographic variables on the development of psychopathology has also been widely studied (Altemus, Sarvaiya, \& Epperson, 2014; Tang, Liu, Liu, Xue, \& Zhang, 2014). Despite some authors consider that their importance could be explained in terms of their relationship to the amount of personal and social resources the individual has, or perceives to have (González, Fernández, Perez, \& Friend, 2007).

In conclusion, the results of this research support the studied variables as vulnerability factors for mental health (anxiety and depressive symptoms) on both general and clinical samples (AD in particular), although with different weights depending on the sample considered.

The clinical implications are evident. Coping resources and personality, with some sociodemographic characteristics and perceived stress, should be considered in the assessment and treatment of those people exposed to situations of significant stress. These variables seem to make up important aspects in the $\mathrm{AD}$ diagnosis and treatment, but they may be even more relevant in people who have not yet developed mental health problems.

This study is not without its limitations. It is a crosssectional and correlational study; therefore, causality re- lations cannot be established. Only retrospective selfreports with the implied bias were used, meaning that the psychopathology in itself could have had some influence on the person's self-perception or self-description (Costa, Bagby, Herbst, \& McCrae, 2005). On the other hand, the mediating or moderating role of some variables was not analysed, since they exceeded the objectives of this study and the size of the sample was insufficient. Finally, other variables related to stress that may have influenced the results, like the controllability and predictability of the stressors (Sandín, 2008), have not been studied. 